\title{
The Quality of Colonoscopy Reporting in Usual Practice: Are Endoscopists Reporting Key Data Elements?
}

\author{
S. D. Hadlock, ${ }^{1}$ N. Liu, ${ }^{2}$ M. Bernstein, ${ }^{3}$ M. Gould, ${ }^{4}$ L. Rabeneck, ${ }^{2,5,6,7,8}$ \\ A. Ruco, ${ }^{3}$ R. Sutradhar, ${ }^{2,8}$ and J. M. Tinmouth ${ }^{2,3,5,6,7}$ \\ ${ }^{1}$ Montfort Hospital, Gastroenterology, Ottawa, ON, Canada K1K 0T2 \\ ${ }^{2}$ Institute for Clinical Evaluative Sciences, Toronto, ON, Canada M4N 3M5 \\ ${ }^{3}$ Sunnybrook Health Sciences Centre, Toronto, ON, Canada M4N 3M5 \\ ${ }^{4}$ Toronto Digestive Disease Associates Inc, Toronto, ON, Canada L4L 4 Y7 \\ ${ }^{5}$ Cancer Care Ontario, Toronto, ON, Canada M5G 2 L7 \\ ${ }^{6}$ Department of Medicine, University of Toronto, Toronto, ON, Canada M5S 1A8 \\ ${ }^{7}$ Institute of Health Policy, Management and Evaluation, University of Toronto, Toronto, ON, Canada M5T 3M6 \\ ${ }^{8}$ Dalla Lana School of Public Health, University of Toronto, Toronto, ON, Canada M5T 3M7
}

Correspondence should be addressed to J. M. Tinmouth; jill.tinmouth@sunnybrook.ca

Received 19 July 2015; Accepted 3 July 2016

Academic Editor: John Marshall

Copyright (C) 2016 S. D. Hadlock et al. This is an open access article distributed under the Creative Commons Attribution License, which permits unrestricted use, distribution, and reproduction in any medium, provided the original work is properly cited.

\begin{abstract}
Background. High quality reporting of endoscopic procedures is critical to the implementation of colonoscopy quality assurance programs. Objective. The aim of our research was to (1) determine the quality of colonoscopy (CS) reporting in "usual practice," (2) identify factors associated with good quality reporting, and (3) compare CS reporting in open-access and non-open-access procedures. Methods. 557 CS reports were randomly selected and assigned a score based on the number of mandatory data elements included in the report. Reports documenting greater than $70 \%$ of the mandatory data elements were considered to be of good quality. Physician and procedure factors associated with good quality CS reporting were identified. Results. Variables that were consistently well documented included date of the procedure (99.6\%), procedure indication (88.9\%), a description of the most proximal anatomical segment reached (98.6\%), and documentation of polyp location (97.8\%). Approximately $79.4 \%$ of the reports were considered to be of good quality. Gastroenterology specialty, lower annual CS volume, and fewer years in practice were associated with good quality reporting. Discussion. CS reporting in usual practice in Ontario lacks quality in several areas. Almost 1 in 5 reports was of poor quality in our study. Conclusions. Targeted interventions and/or use of mandatory fields in synoptic reports should be considered to improve CS reporting.
\end{abstract}

\section{Introduction}

Colonoscopy (CS) is an important tool used to screen for cancerous and precancerous lesions of the colon. With increasing public awareness of the need for colorectal cancer (CRC) screening, the use of CS has been steadily rising [1]. Increasing use of CS, particularly in the context of organized CRC screening programs, has contributed to a growing interest in improving the quality of endoscopic procedures and measuring specific quality indicators [2-4].
Until recently, the lack of standardized accepted approach to CS reporting was a significant obstacle to measuring these quality indicators. Furthermore, the CS report is also the primary medicolegal documentation as it provides a detailed description of the procedure performed [5]. Open-access (OA) procedures, where the patient is seen directly for CS without a recent office visit with the endoscopist performing the procedure, are increasingly being done [6], which may require more extensive documentation to sufficiently capture all pertinent information. Finally, the quality of the CS 
report may have a significant impact on clinical care as inadequate documentation may result in needlessly repeating procedures, exposing patients to unnecessary risks.

In 1999, the American Society for Gastrointestinal Endoscopy published guidelines for quality improvement of endoscopy [7]. More recently, the Quality Assurance Task Force of the National Colorectal Cancer Roundtable (NCCRT) has developed a data reporting system for colonoscopy (CO-RADS), which outlines the essential elements to include in each CS report and highlights key indicators for quality improvement [8]. However, despite the recent interest in measuring the quality of CS reporting, only a limited number of studies have addressed this important issue [9-20]. Most prior work has been limited to single academic institutions and/or certain populations $[9,11,13-$ $20]$, computer generated reporting $[10,11,13,15,16,19]$, or intervention and/or audit studies [13, 16-20]. These factors have limited the generalizability of prior studies. The aims of our research were to (1) determine the quality of CS reporting in clinical practice using established CS reporting guidelines; (2) identify factors associated with good quality CS reporting; and (3) compare CS reporting in OA and non-open-access (NOA) procedures.

\section{Methods}

2.1. Data Sources. This study evaluated CS reports that were obtained from a larger parent study that was designed to validate colonoscopy data elements in Ontario's health administrative databases using a large scale population-based chart review. These datasets, held at the Institute for Clinical Evaluative Sciences (ICES) and the Canadian Institute for Health Information (CIHI), are linked using unique, encoded identifiers and analyzed at ICES. Using a stratified sampling strategy, the parent study randomly selected 1,400 CS procedures from fiscal year (FY) 2008 from 30 randomly selected facilities (24 hospitals and 6 nonhospital endoscopy clinics) in Ontario using the Ontario Health Insurance Plan (OHIP) Claims History Database, the National Ambulatory Care Reporting System (NACRS), and the CIHI-Discharge Abstract Database (DAD).

From the larger parent study, a convenience sample of 575 reports was randomly selected for analysis in the current study as reabstraction of reports was required to evaluate quality of CS reporting. Report formats ranged from hand-written to dictated notes to proprietary or local software with or without electronic medical record (EMR). All personal health information, including endoscopist name, were removed from the reports prior to review. Each report was identified by date of procedure and the ICES key number (IKN), which is an encrypted version of the provincial health insurance plan number. Using the IKN, the data abstracted from each report were linked to the Ontario health administrative databases in order to analyze physician and procedure factors associated with good CS reporting.

Ethics approval for this study was obtained from the Sunnybrook Health Sciences Centre Research Ethics Board.
TABLE 1: Number and proportion of mandatory data elements documented among CS reports $(N=557)$.

\begin{tabular}{lcc}
\hline Mandatory data element & Documented, $n(\%)$ & $\begin{array}{c}\text { Total number } \\
\text { of reports }\end{array}$ \\
\hline $\begin{array}{l}\text { Preprocedure elements } \\
\text { Indication }\end{array}$ & $495(88.9)$ & 557 \\
\hline $\begin{array}{l}\text { Procedure elements } \\
\text { Date of procedure }\end{array}$ & $555(99.6)$ & 557 \\
Sedation: name & $364(65.4)$ & 557 \\
Sedation: dose & $318(57.1)$ & 557 \\
Anatomical segment reached & $549(98.6)$ & 557 \\
Cecal/TI landmarks & $287(67.4)$ & 426 \\
Quality of bowel preparation & $192(34.5)$ & 557 \\
Colonic mass: location & $16(100)$ & 16 \\
Colonic mass: size & $11(68.8)$ & 16 \\
Colonic polyp (1st): location & $177(97.8)$ & 181 \\
Colonic polyp (1st): size & $83(45.9)$ & 181 \\
\hline Postprocedure elements & & \\
Summary statement & $425(76.3)$ & 557 \\
Management plan & $438(78.6)$ & 557 \\
\hline
\end{tabular}

CS: colonoscopy; TI: terminal ileum.

2.2. Evaluation of CS Report Quality. We convened a panel of endoscopists with expertise on the topic (JT, SH, LR, $\mathrm{MG}$, and $\mathrm{MB}$ ) prior to evaluating the CS reports. The objective of the panel was to decide on the mandatory quality indicators required for good quality CS reporting. The panel reviewed the 1999 ASGE guidelines [7] and the 2007 NCCRT recommendations for CS reporting [8]. All data elements recommended by these reports (preprocedure, procedure, and postprocedure) as necessary for high quality CS reporting were reviewed by the panel. The panel then identified 33 data elements to be used to evaluate the quality of CS reporting in our sample based on existing guidelines. Of these, the panel categorized 13 data elements as "mandatory" (Table 1) based on their perceived importance. These include indication and date of the procedure, sedation name and dose, anatomical segment reached, cecal/terminal ileum (TI) landmarks through written indication, quality of bowel preparation, location and size of any mass found, location and size of any polyp found, summary statement, and management plan. Each CS report was assigned a score based on the number of mandatory data elements included in the report (1 point for each data element). Generally, elements were categorized as nonmandatory if it was likely that they would be captured in the documentation of the preendoscopy consultation visit.

2.3. Physician and Procedure Factors Analyzed. Using the databases housed at ICES, we collected information on the institution (teaching/community hospital or private clinic) where the CS was performed, whether it was an OA procedure (patient seen directly for CS without an office visit with the endoscopist performing the procedure in the prior 
5 years), and physician factors. Physician factors included specialty (gastroenterology, surgery, internal medicine, and general practitioner/other), number of years in practice (number of years since graduation from medical school), annual CS volume (mean annual number of procedures completed in the preceding year), CS completion rate (proportion of successful cecal/TI intubations in the preceding year), and polypectomy rate (proportion of CS procedures in the preceding year where at least one polyp was detected).

2.4. Statistical Analysis. CS reports were evaluated using a standardized data collection form. Reports were assigned a score from 0 to a maximum of 13 , with each mandatory data element representing one point. The number and proportion of CS reports complying with each mandatory data element were recorded. Reports documenting $\geq 70 \%$ of the possible mandatory data elements were classified as "good" quality reports and the remaining reports were classified as "poor" quality based on a similar threshold used in previous work [9]. For this calculation, the denominator varied depending on the number of measurable data elements (e.g., if there were no polyps or masses, related data elements were not included in the denominator for that specific report). In analyzing the quality of CS reporting in OA procedures, we also evaluated the documentation of nonmandatory data elements. As OA procedures were not preceded by a consultation visit, it was assumed that reports from these procedures should be more comprehensive than the reports from NOA procedures.

Univariate and multivariable logistic regression were used to model physician and institutional factors (institution; OA status; physician specialty; years in practice; annual CS volume; polypectomy rate; CS completion rate) associated with the binary outcome of good quality CS reporting. A generalized estimating equations approach was implemented to account for clustering of colonoscopies within physicians [21]. All analyses were conducted using SAS v.9 statistical software (SAS Institute, Cary, NC).

\section{Results}

Five hundred and seventy-five CS reports ( 495 from hospitals and 80 from private clinics) were evaluated. Reports by physicians missing information on annual CS volume were excluded from further analyses $(n=18)$. Colonoscopies were performed by 114 physicians at 15 sites (10 hospitals and 5 private clinics) in Ontario. Each physician and each site contributed from 1 to 25 colonoscopies and from 9 to 113 reports to the sample, respectively.

Documentation of the mandatory data elements is reported in Table 1. Variables that were consistently well documented included date of the procedure $(99.6 \%)$, procedure indication $(88.9 \%)$, a description of the most proximal anatomical segment reached (98.6\%), and documentation of polyp location (97.8\%). By contrast, the quality of bowel preparation (34.5\%) and polyp size (45.9\%) were documented in less than half of all reports. Over $20 \%$ of reports did not include a summary statement or follow-up plan. When the reports were assigned a score representing the overall completeness of documentation,

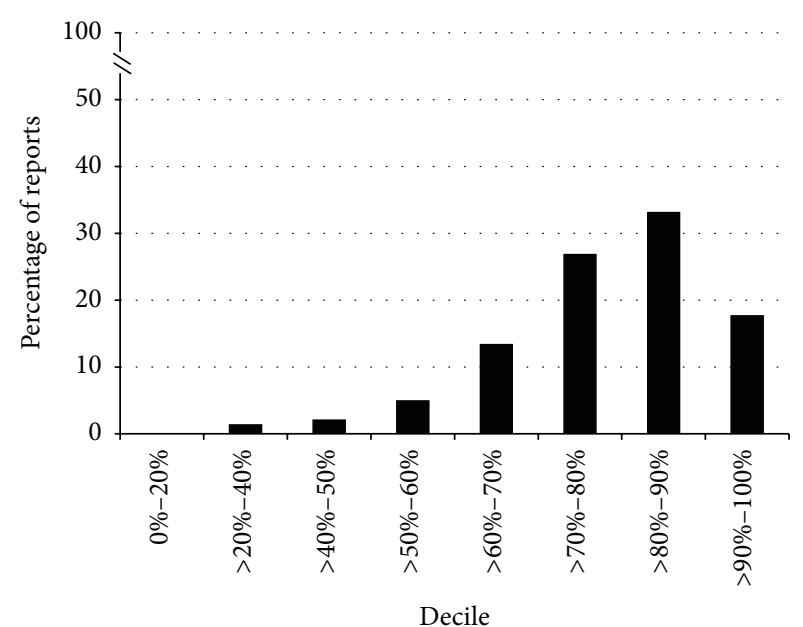

FIGURE 1: Distribution of the reports by decile of mandatory data elements reported, from 0 to $100 \%$.

$442(79.4 \%)$ of the reports were considered good quality (documenting $\geq 70 \%$ of the mandatory indicators). Figure 1 depicts the distribution of the reports in each decile of completeness, ranging from 0 to $100 \%$ of possible mandatory elements. Nonmandatory data elements reported by decile are presented in Supplementary Materials available online at http://dx.doi.org/10.1155/2016/1929361.

Univariate analyses demonstrated that the quality of reporting varied significantly at the physician level for specialty, number of years in practice, CS volume, CS completion rate, and institution type (Table 2). Of the variables included in the multivariate analysis, surgeons and internists were significantly less likely to have good quality reporting compared to gastroenterologists (OR 0.19 (95\% CI 0.11-0.34) for surgeons and 0.22 (95\% CI 0.09-0.53) for internists) (Table 3). For every additional year in practice, there was a $5 \%$ decrease in the odds of good quality reporting. Physicians with higher annual CS volume practices were also less likely to have good quality reporting (OR 0.86 per 100 procedures (95\% CI 0.76-0.97)).

Compared to NOA procedures, OA procedures were not associated with good quality of reporting on mandatory data elements in the multivariable analysis. However, several nonmandatory data elements, patient history, family history, comorbidity, and physical exam, were consistently reported to a greater extent in OA procedures compared to NOA procedures (Table 4). Aside from sedation name, which was only documented in $48.0 \%$ of OA procedures compared to $70.4 \%$ of NOA procedures, OA reports consistently documented all quality indicators to a similar extent or better compared to NOA reports. In general, whether OA or NOA procedure, nonmandatory data elements were documented less well than mandatory elements.

\section{Discussion}

Our study of CS reporting in usual practice in Ontario reveals that 1 in 5 reports fail to document key quality 
TABLE 2: Physician and institution factors associated with good quality CS reporting.

\begin{tabular}{|c|c|c|c|c|c|}
\hline Variable & Value & Good quality $(n=442)$ & Poor quality $(n=115)$ & Total $(N=557)$ & $P$ value \\
\hline \multirow{2}{*}{ Completion rate, $n(\%)$} & $<90 \%$ & $162(36.7)$ & $38(33.0)$ & $200(35.9)$ & \multirow{2}{*}{0.006} \\
\hline & $\geq 90 \%$ & $280(63.3)$ & $77(67.0)$ & $357(64.1)$ & \\
\hline \multirow{2}{*}{ Open-access CS, $n(\%)$} & No & $94(21.3)$ & $31(27.0)$ & $125(22.4)$ & \multirow{2}{*}{ NS } \\
\hline & Yes & $348(78.7)$ & $84(73.0)$ & $432(77.6)$ & \\
\hline \multirow{2}{*}{ Polypectomy rate } & Mean \pm SD & $0.22 \pm 0.10$ & $0.21 \pm 0.09$ & $0.22 \pm 0.09$ & \multirow{2}{*}{ NS } \\
\hline & Median (IQR) & $0.20(0.14-0.28)$ & $0.20(0.16-0.24)$ & $0.20(0.15-0.27)$ & \\
\hline \multirow{2}{*}{ Annual CS volume } & Mean \pm SD & $429.17 \pm 254.25$ & $578.97 \pm 372.99$ & $460.10 \pm 288.93$ & \multirow{2}{*}{$<0.001$} \\
\hline & Median (IQR) & $443(201-557)$ & $535(280-752)$ & $465(202-588)$ & \\
\hline \multirow{2}{*}{ Institution type, $n(\%)$} & Clinic & $51(11.5)$ & $25(21.7)$ & $76(13.6)$ & \multirow{2}{*}{0.005} \\
\hline & Hospital & $391(88.5)$ & $90(78.3)$ & $491(88.2)$ & \\
\hline \multirow{3}{*}{ Physician specialty, $n(\%)$} & Surgeon & $205(46.4)$ & $75(65.2)$ & $280(50.3)$ & \multirow{3}{*}{$<0.001$} \\
\hline & Internist & $34(7.7)$ & $17(14.8)$ & $51(9.2)$ & \\
\hline & GI & $203(45.9)$ & $23(20.0)$ & $226(40.6)$ & \\
\hline \multirow{2}{*}{ Years in practice } & Mean \pm SD & $23.10 \pm 12.00$ & $28.85 \pm 11.28$ & $24.28 \pm 12.07$ & \multirow{2}{*}{$<0.001$} \\
\hline & Median (IQR) & $21(14-30)$ & $28(20-39)$ & $23(15-31)$ & \\
\hline
\end{tabular}

CS: colonoscopy; GI: gastroenterologist.

TABLE 3: Multivariable analysis on factors associated with good quality CS reporting.

\begin{tabular}{|c|c|c|}
\hline Variable & OR $(95 \% \mathrm{CI})$ & $P$ value \\
\hline \multicolumn{3}{|l|}{ Completion rate, $\%$} \\
\hline$<90 \%$ & $0.92(0.56-1.52)$ & \multirow{2}{*}{ NS } \\
\hline$\geq 90 \%$ & 1.0 & \\
\hline \multicolumn{3}{|l|}{ Open-access CS } \\
\hline No & $1.12(0.64-1.96)$ & \multirow{2}{*}{ NS } \\
\hline Yes & 1.0 & \\
\hline \multicolumn{3}{|l|}{ Polypectomy rate, \% } \\
\hline Per $1 \%$ & $0.42(0.03-5.17)$ & NS \\
\hline \multicolumn{3}{|l|}{ Annual CS volume } \\
\hline Per 100 procedures & $0.86(0.76-0.97)$ & 0.01 \\
\hline \multicolumn{3}{|l|}{ Institution type } \\
\hline Clinic & $1.36(0.46-3.99)$ & \multirow{2}{*}{ NS } \\
\hline Hospital & 1.0 & \\
\hline \multicolumn{3}{|l|}{ Physician specialty } \\
\hline Surgeon & $0.19(0.11-0.34)$ & $<0.0001$ \\
\hline Internist & $0.22(0.09-0.53)$ & 0.0008 \\
\hline GI & 1.0 & \\
\hline \multicolumn{3}{|l|}{ Years in practice, years } \\
\hline Per year & $0.95(0.93-0.97)$ & $<0.0001$ \\
\hline
\end{tabular}

CS: colonoscopy; GI: gastroenterologist.

indicators. Good quality CS reporting is associated with gastroenterology specialty, physicians with fewer years in clinical practice, and those with lower CS volumes.

The importance of our study is that it is the first to evaluate the quality of CS reporting in usual clinical practice, given the random sampling method that was used to identify study participants. Previous published studies have been limited in their generalizability for numerous reasons. First, prior work has been limited to single institutions or homogenous populations [9, 11, 13-20]. For example, Palmer et al. [11] reported on the quality of 135 CS reports of communitybased physicians but this study was limited to a cohort of patients from a single Veterans Administration (VA) Medical Centre. The homogeneous patient population and restricted geographic variation of the participating physicians limit the applicability of the results to larger population-based practices. Second, CS reports evaluated in previous work have been computer-generated $[10,11,13,15,16,19]$, which may not be representative of other jurisdictions [22-25]. Finally, previous reported results have been of audit or intervention studies, where physicians were aware that they were being assessed [13, 16-20].

Prior published studies have reported similar findings to ours. For example, in 2002, Robertson et al. [9] evaluated a single CS report from 122 endoscopy centres in the United States using the 1999 ASGE reporting guidelines as the reference standard. The authors found that the quality of CS reporting was highly variable with indication for the procedure, examination extent, and polyp location and removal being well documented [9]. On the other hand, patient history, informed consent, medications used during the procedure, and adequacy of bowel preparation were poorly documented [9]. The largest study, published by Lieberman et al. [10] in 2009, reviewed 438,521 computer generated reports from the Clinical Outcomes Research Initiative (CORI) database. This study demonstrated higher documentation rates than previously reported but did note considerable variation in the quality of reporting across the 73 practice sites, despite using a standardized computer based reporting tool. More recently, a study examining 4,800 computerized CS reports from 12 endoscopy departments in the Netherlands showed variation among quality indicators with withdrawal time, polyp size, polyp morphology, and quality of bowel preparation being of poorer compliance with reporting among the 117 endoscopists [13]. Singh et 
TABLE 4: Number and proportion of selected* nonmandatory data elements documented among CS reports by open-access (OA) status.

\begin{tabular}{|c|c|c|c|c|}
\hline Variable & Value & $\begin{array}{l}\text { Open access }(\mathrm{OA}) \\
(n=125)\end{array}$ & $\begin{array}{c}\text { Nonopen access } \\
\text { (NOA) } \\
(n=432)\end{array}$ & Total $(N=557)$ \\
\hline \multirow{2}{*}{ History, $n(\%)$} & Yes & $79(63.2)$ & $99(22.9)$ & $178(32.0)$ \\
\hline & No & $46(36.8)$ & $333(77.1)$ & $379(68.0)$ \\
\hline \multirow{2}{*}{ Comorbidity, $n(\%)$} & Yes & $75(60.0)$ & $60(13.9)$ & $135(24.2)$ \\
\hline & No & $50(40.0)$ & $372(86.1)$ & $422(75.8)$ \\
\hline \multirow{2}{*}{ Physical exam, $n(\%)$} & Yes & $57(45.6)$ & $67(15.5)$ & $124(22.3)$ \\
\hline & No & $68(54.4)$ & $365(84.5)$ & $433(77.7)$ \\
\hline \multirow{2}{*}{ Medication list, $n(\%)$} & Yes & $66(52.8)$ & $45(10.4)$ & $111(19.9)$ \\
\hline & No & $59(47.2)$ & $387(89.6)$ & $446(80.1)$ \\
\hline \multirow{2}{*}{ Informed consent, ${ }^{\mathrm{a}} n(\%)$} & Yes & $58(46.4)$ & $93(21.5)$ & $151(27.1)$ \\
\hline & No & $67(53.6)$ & $339(78.5)$ & $406(72.9)$ \\
\hline \multirow{2}{*}{ Family history, $n(\%)$} & Yes & $76(60.8)$ & $74(17.1)$ & $150(26.9)$ \\
\hline & No & $49(39.2)$ & $358(82.9)$ & $407(73.1)$ \\
\hline \multirow{2}{*}{$\begin{array}{l}\text { History of previous/no previous CS } \\
\text { noted, } n(\%)\end{array}$} & Yes & $31(24.8)$ & $61(14.1)$ & $92(16.5)$ \\
\hline & No & $94(75.2)$ & $371(85.9)$ & $465(83.5)$ \\
\hline \multirow{2}{*}{ Year of previous CS, ${ }^{\mathrm{b}} n(\%)$} & Yes & $21(67.7)$ & $36(59.0)$ & $57(62.0)$ \\
\hline & No & $10(32.3)$ & $25(41.0)$ & $35(38.0)$ \\
\hline \multirow{2}{*}{$\begin{array}{l}\text { Adequate information to determine if } \\
\text { CS interval is appropriate, }{ }^{c} n(\%)\end{array}$} & Yes & $13(56.5)$ & $20(35.1)$ & $33(41.3)$ \\
\hline & No & $10(43.5)$ & $37(64.9)$ & $47(58.8)$ \\
\hline \multirow{2}{*}{$\begin{array}{l}\text { Retroflexion/no retroflexion noted, } n \\
(\%)\end{array}$} & Yes & $23(18.4)$ & $96(22.2)$ & $119(21.4)$ \\
\hline & No & $102(81.6)$ & $336(77.8)$ & $438(78.6)$ \\
\hline
\end{tabular}

NOA: nonopen access; OA: open access.

${ }^{*}$ Restricted to 10 data elements (from 20 assessed), largely because small cell sizes required suppression of the data.

${ }^{a}$ Excluding 12 reports with missing data element.

${ }^{\mathrm{b}}$ Only valid for the 92 who provided information on prior colonoscopy history.

${ }^{\mathrm{c}}$ Only valid for the 80 who provided a valid response.

al. [12] recently reported on a sample of 797 CS reports from six community and academic hospitals in Manitoba, Canada. Approximately $80 \%$ of the reports did not include information on the quality of bowel preparation and only $34 \%$ of the reports contained information on polyp morphology [12].

Reporting on the quality of bowel preparation was consistently low (34.5\%) in our study and in prior work $[9,12$, 13]. One outlier was the study by Beaulieu et al. [15] that examined 250 consecutive computerized endoscopic reports from a tertiary care institution in Quebec, Canada, using the indicators set forth by the Quality Assurance Task Group of the NCCRT. The quality of bowel preparation in this sample was recorded in $99.9 \%$ of the reports [15]. The high compliance with reporting in the study by Beaulieu et al. [15] may be a result of using a computerized report generator with compulsory fields, using preformatted text or drop-down menus, or may reflect local practice patterns as all procedures were completed at a single institution. Without a compulsory field for bowel preparation, physicians may assume that they only need to report it when bowel preparation is insufficient.

Several physician factors were associated with good quality CS reporting. Our findings demonstrated higher quality reporting by gastroenterologists and physicians with lower annual CS volumes. One possible explanation is that physicians performing fewer colonoscopies may take more time for procedure documentation. Physicians with fewer years in clinical practice were also found to have better reporting. This finding may reflect the increasing emphasis on clinical documentation and quality assurance in recent medical education or, simply, that, over time, physicians generate less detailed reports that are of poorer quality. Our results identify a number of target groups for quality improvement initiatives; for example, surgical training programs should ensure that residents are aware of the importance and key elements of good endoscopy reporting. Further, individual endoscopy units may also benefit from an audit and feedback tool to assess their CS reporting quality.

Documentation in OA procedures is particularly important as patients are not seen in a prior consultation where information on the patient, medications, and family history would be expected to be documented. However, exploring quality of CS reporting among OA and NOA procedures revealed that several nonmandatory indicators were consistently reported to a greater extent in OA procedures. These included patient history, family history, comorbidity, medication list, and history of previous CS examinations. Although unsurprising as patients attending NOA procedures had been 
seen in consultation beforehand and nonmandatory elements were likely recorded at that visit, reporting of OA procedures was still suboptimal as a third or more of these reports were missing data elements such as history of presenting illness, comorbidity, and medications.

Our results must be interpreted in consideration of the strengths and limitations of the study. We included data from a large random sample of institutions on CS performed by a large random sample of physicians across Ontario, increasing the generalizability of our findings. Furthermore, our study population was comprised primarily of physicians using nonstandardized CS reporting tools (non-EMR) and is representative of usual practice in many other jurisdictions [25]. Unlike the largest studies limited to EMR/software or single centres, our design captured heterogeneity in usual practice across multiple sites by including various report formats and sources. As others have shown [10, 13], there is important variation even with electronic reporting systems; our results can be used to inform the design of such systems to ensure high quality reports. Limitations include the use of an arbitrary threshold of $70 \%$ for determining good quality reporting. However, previous work [9] has also employed a similar threshold. Our findings are not intended to address the quality of the included procedures nor whether included elements were reported correctly; rather our goal was to measure how well endoscopists completed the CS report. The CS report is the most comprehensive description of the procedure and is commonly used to assess the quality of the procedure; reports that are missing key elements will undermine quality improvement initiatives. Lastly, the study does rely in part on accurate entry of billing codes by physicians; however this information was used only to determine which procedure and physician factors were associated with good quality reporting and not to assess the quality of the reports themselves.

In conclusion, CS reporting in usual practice in Ontario lacks quality in several areas. Almost 1 in 5 reports were of poor quality in our study, and bowel prep quality and polyp size were documented in less than half of reports. Targeted interventions and/or use of mandatory fields in synoptic reports should be considered to improve CS reporting.

\section{Disclosure}

The funding source had no involvement in the study design, collection, and analysis of data, in the writing of the report, or in the decision to submit the paper for publication. The opinions, results, and conclusions reported in this paper are those of the authors and are independent from the funding sources. No endorsement by ICES or the Ontario MOHLTC is intended or should be inferred. Parts of this material are based on data and information compiled and provided by CIHI. However, the analyses, conclusions, opinions, and statements expressed herein are those of the authors and not necessarily those of CIHI.

\section{Competing Interests}

The authors declare that they have no competing interests.

\section{Acknowledgments}

This study was funded by a grant from the Canadian Cancer Society Research Institute (no. 20332). This study was supported by the Institute for Clinical Evaluative Sciences (ICES), which is funded by an annual grant from the Ontario Ministry of Health and Long-Term Care (MOHLTC).

\section{References}

[1] C. Vinden, S. Schultz, and L. Rabeneck, ICES Research Atlas: Use of Large Bowel Procedures in Ontario, Institute for Clinical Evaluative Sciences, 2004.

[2] M. F. Kaminski, J. Regula, E. Kraszewska et al., "Quality indicators for colonoscopy and the risk of interval cancer," The New England Journal of Medicine, vol. 362, no. 19, pp. 1795-1803, 2010.

[3] R. L. Barclay, J. J. Vicari, A. S. Doughty, J. F. Johanson, and R. L. Greenlaw, "Colonoscopic withdrawal times and adenoma detection during screening colonoscopy," New England Journal of Medicine, vol. 355, no. 24, pp. 2533-2541, 2006.

[4] D. K. Rex, J. L. Petrini, T. H. Baron et al., "Quality indicators for colonoscopy," The American Journal of Gastroenterology, vol. 101, no. 4, pp. 873-885, 2006.

[5] D. K. Rex, "Avoiding and defending malpractice suits for postcolonoscopy cancer: advice from an expert witness," Clinical Gastroenterology and Hepatology, vol. 11, no. 7, pp. 768-773, 2013.

[6] S. Hadlock, L. Rabeneck, L. F. Paszat, R. Sutradhar, A. S. Wilton, and J. Tinmouth, "Open-access colonoscopy in Ontario: associated factors and quality," Canadian Journal of Gastroenterology, vol. 27, no. 6, pp. 341-346, 2013.

[7] American Society for Gastrointestinal Endoscopy, "Quality improvement of gastrointestinal endoscopy: guidelines for clinical application. From the ASGE," Gastrointestinal Endoscopy, vol. 49, no. 6, pp. 842-844, 1999.

[8] D. Lieberman, M. Nadel, R. A. Smith et al., "Standardized colonoscopy reporting and data system: report of the Quality Assurance Task Group of the National Colorectal Cancer Roundtable," Gastrointestinal Endoscopy, vol. 65, no. 6, pp. 757766, 2007.

[9] D. J. Robertson, L. B. Lawrence, N. J. Shaheen et al., "Quality of colonoscopy reporting: a process of care study," The American Journal of Gastroenterology, vol. 97, no. 10, pp. 2651-2656, 2002.

[10] D. A. Lieberman, D. O. Faigel, J. R. Logan et al., "Assessment of the quality of colonoscopy reports: results from a multicenter consortium," Gastrointestinal Endoscopy, vol. 69, no. 3, pp. 645653, 2009.

[11] L. B. Palmer, D. H. Abbott, N. Hamilton, D. Provenzale, and D. A. Fisher, "Quality of colonoscopy reporting in community practice," Gastrointestinal Endoscopy, vol. 72, no. 2, pp. 321-327, 2010.

[12] H. Singh, L. Kaita, G. Taylor, Z. Nugent, and C. Bernstein, "Practice and documentation of performance of colonoscopy in a central Canadian health region," Canadian Journal of Gastroenterology and Hepatology, vol. 28, no. 4, pp. 185-190, 2014.

[13] V. de Jonge, J. Sint Nicolaas, D. L. Cahen et al., "Quality evaluation of colonoscopy reporting and colonoscopy performance in daily clinical practice," Gastrointestinal Endoscopy, vol. 75, no. 1, pp. 98-106, 2012. 
[14] J. Li, M. R. Nadel, C. F. Poppell, D. M. Dwyer, D. A. Lieberman, and E. K. Steinberger, "Quality assessment of colonoscopy reporting: results from a statewide cancer screening program," Diagnostic and Therapeutic Endoscopy, vol. 2010, Article ID 419796, 7 pages, 2010.

[15] D. Beaulieu, A. Barkun, and M. Martel, "Quality audit of colonoscopy reports amongst patients screened or surveilled for colorectal neoplasia," World Journal of Gastroenterology, vol. 18, no. 27, pp. 3551-3557, 2012.

[16] D. Beaulieu, M. Martel, and A. Barkun, "A prospective intervention study of colonoscopy reporting among patients screened or surveilled for colorectal neoplasia," Canadian Journal of Gastroenterology, vol. 26, no. 10, pp. 718-722, 2012.

[17] H. D. Mai, R. A. Sanowski, and J. P. Waring, "Improved patient care using the A/S/G/E guidelines on quality assurance: a prospective comparative study," Gastrointestinal Endoscopy, vol. 37, no. 6, pp. 597-599, 1991.

[18] S. G. Coe, C. Panjala, M. G. Heckman et al., "Quality in colonoscopy reporting: an assessment of compliance and performance improvement," Digestive and Liver Disease, vol. 44, no. 8, pp. 660-664, 2012.

[19] S. C. van Doorn, J. van Vliet, P. Fockens, and E. Dekker, "A novel colonoscopy reporting system enabling quality assurance," Endoscopy, vol. 46, no. 3, pp. 181-187, 2014.

[20] S. G. Coe, J. E. Crook, N. N. Diehl, and M. B. Wallace, "An endoscopic quality improvement program improves detection of colorectal adenomas," The American Journal of Gastroenterology, vol. 108, no. 2, pp. 219-226, 2013.

[21] K. Y. Liang and S. L. Zeger, "Longitudinal data analysis using generalized linear models," Biometrika, vol. 73, no. 1, pp. 13-22, 1986.

[22] R. Birtwhistle and T. Williamson, "Primary care electronic medical records: a new data source for research in Canada," Canadian Medical Association Journal, vol. 187, no. 4, pp. 239240, 2015.

[23] J. A. Linder, J. Ma, D. W. Bates, B. Middleton, and R. S. Stafford, "Electronic health record use and the quality of ambulatory care in the United States," Archives of Internal Medicine, vol. 167, no. 13, pp. 1400-1405, 2007.

[24] L. Zhou, C. S. Soran, C. A. Jenter et al., "The relationship between electronic health record use and quality of care over time," Journal of the American Medical Informatics Association, vol. 16, no. 4, pp. 457-464, 2009.

[25] C. M. DesRoches, E. G. Campbell, S. R. Rao et al., "Electronic health records in ambulatory care-a national survey of physicians," The New England Journal of Medicine, vol. 359, no. 1, pp. 50-60, 2008. 


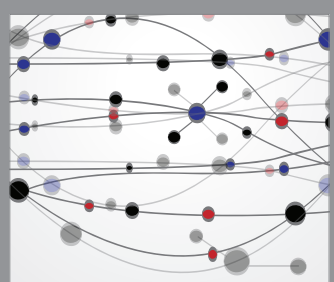

The Scientific World Journal
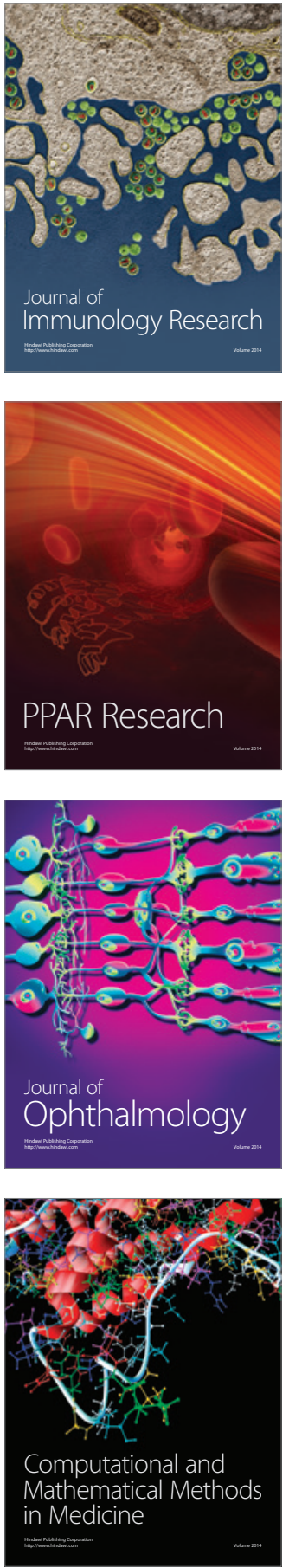

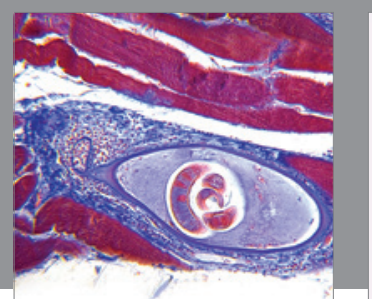

Gastroenterology Research and Practice

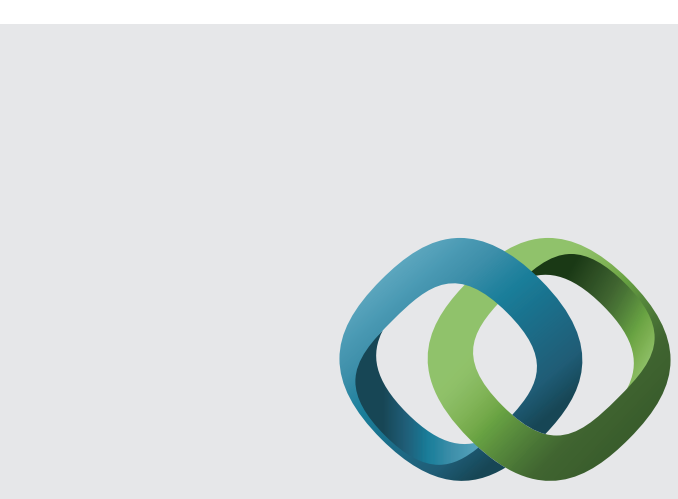

\section{Hindawi}

Submit your manuscripts at

http://www.hindawi.com
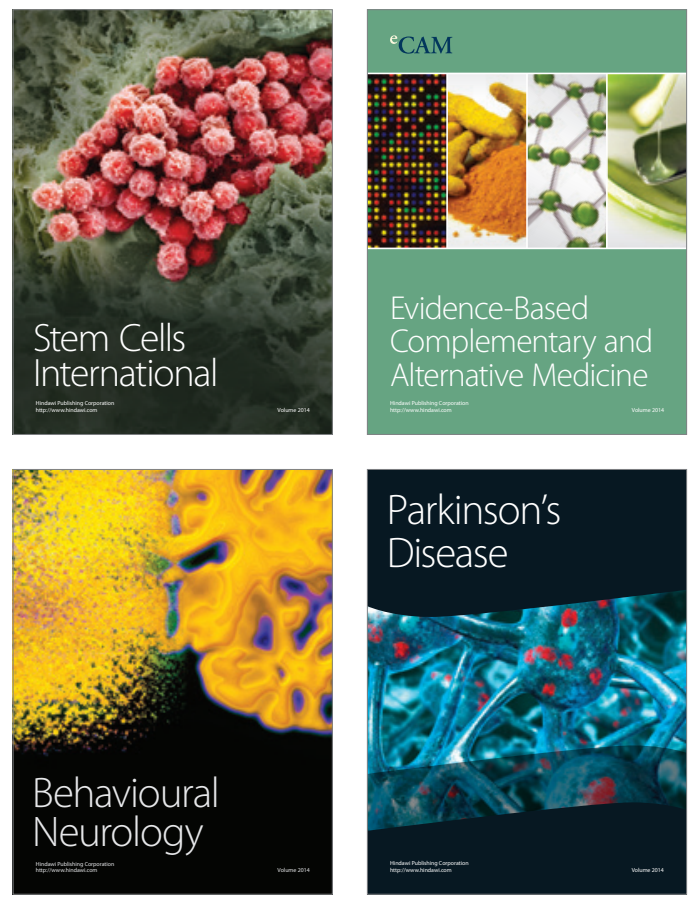
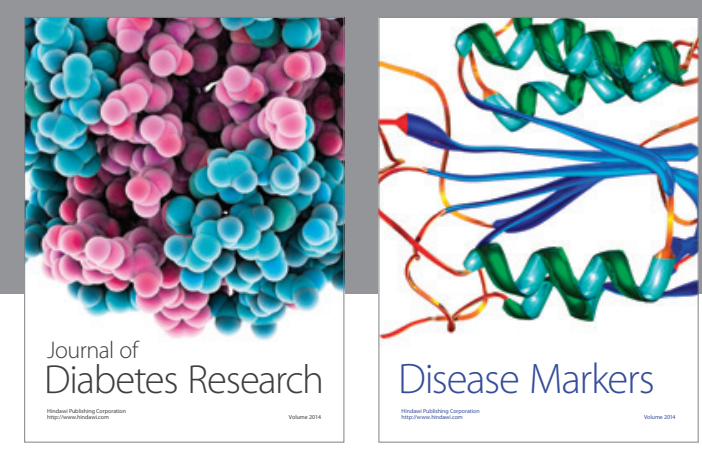

Disease Markers
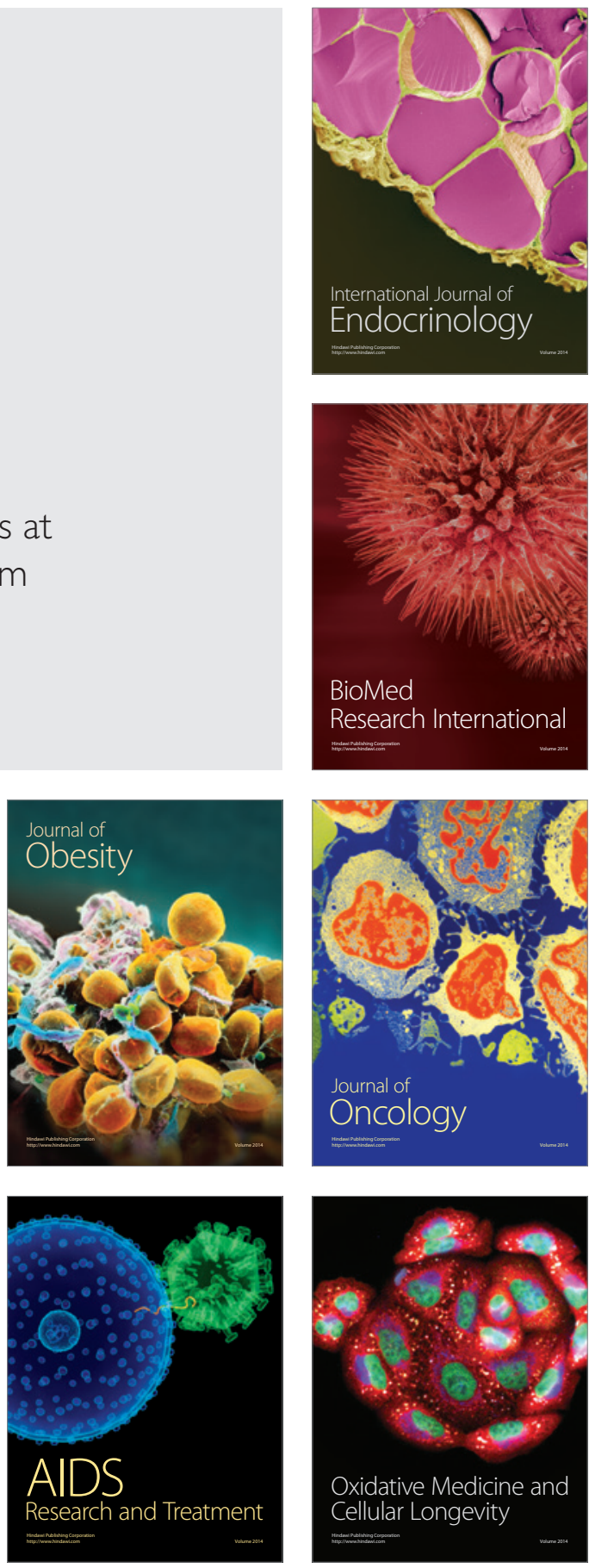